\title{
SUPERVISÃO DE ENFERMAGEM NO USO DE EQUIPAMENTO DE PROTEÇÃO INDIVIDUAL EM UM HOSPITAL GERAL*
}

\author{
Juliana Ferreira de Santana Carvalho', Lucieli Dias Pedreschi Chaves²
}

\begin{abstract}
RESUMO: Este artigo tem como objetivo descrever a supervisão de enfermagem em relação ao uso de Equipamentos de Proteção Individual (EPI), em hospital geral de município no interior paulista. O estudo teve cunho descritivo, sendo a população constituída por 20 enfermeiros, os quais responderam a um questionário. Para análise dos dados foi utilizada estatística descritiva. Os sujeitos, em sua maioria, são mulheres na faixa etária de 20-30 anos e trabalham há menos de três anos no hospital. Quanto ao EPI, $90 \%$ afirmam que a instituição fornece treinamento adequado para seu uso, $95 \%$ referem que existe disponibilidade e para $60 \%$ há resistência da Enfermagem no seu uso, sendo a observação direta (95\%), orientação individual (85\%) e discussão em grupo (80\%) as medidas para a supervisão e estímulo ao uso. O emprego de EPI na prática assistencial constitui um desafio a enfermeiros e supervisores.
\end{abstract}

PALAVRAS CHAVE: Enfermagem; Supervisão de enfermagem; Enfermagem do trabalho.

\section{NURSING SUPERVISION OF THE USE OF PROTECTIVE EQUIPMENT IN A GENERAL HOSPITAL}

\begin{abstract}
This article aims to describe the supervision of nurses in the use of Personal Protective Equipment (PPE), in a general hospital in the municipality of São Paulo. It was a descriptive study, with a population of 20 nurses who answered a questionnaire. Descriptive statistics were used for data analysis. The subjects, most of them were women aged 20-30 years and employed for less than three years in the hospital. As for PPE, 90\% said that the institution provides adequate training for its use, $95 \%$ report that there is availability and there is resistance to $60 \%$ of the Nursing staff in its use. Direct observation (95\%), individual counseling (\% 85) and group discussion (80\%) are the measures for the supervision and encouragement to its use. The use of PPE in the healthcare practice is a challenge for nurses and supervisors.
\end{abstract}

KEYWORDS: Nursing; Nursing Supervisors; Occupational Nursing.

\section{SUPERVISIÓN DE ENFERMERÍA EN USO DE EQUIPO DE PROTECCIÓN INDIVIDUAL EN UN HOSPITAL GENERAL}

RESUMEN: Este artículo tuvo como objetivo describir la supervisión de enfermería acerca del uso de Equipos de Protección Individual (EPI), en hospital general de municipio en interior paulista. El estudio fue descriptivo, siendo su población constituida por 20 enfermeros, los cuales contestaron a un cuestionário. Para análisis de los datos, fue utilizada estadística descriptiva. Los sujetos, en su mayoría, son mujeres en la franja etaria de 20-30 años y trabajan por menos de tres años en hospital. Cuanto al EPI, 90\% afirman que la institución provee entreinamiento adecuado para su uso, $95 \%$ dicen que existe disponibilidad y para $60 \%$ hay resistencia de la Enfermería en su uso, siendo la observación directa (95\%), orientación individual (85\%) y discusión en grupo (80\%) las medidas para la supervisión y estímulo al uso. El empleo de EPI en la práctica asistencial constituye un desafío a enfermeros y supervisores.

PALABRAS CLAVE: Enfermería; Supervisión de enfermería; Enfermería del trabajo.

*Projeto de pesquisa financiado pelo PIC-USP/Santander modalidade iniciação científica, 2008-2009.

${ }^{1}$ Acadêmica da Escola de Enfermagem de Ribeirão Preto da Universidade de São Paulo-EERP-USP.

${ }^{2}$ Enfermeira. Doutora em Enfermagem. Docente do Departamento de Enfermagem Geral e Especializada EERP-USP. Orientadora,

Autor correspondente:

Lucieli Dias Pedreschi Chaves

Escola de Enfermagem de Ribeirão Preto

Av. Bandeirantes, 3900 - 14040-920 - Ribeirão Preto-SP, Brasil

Recebido: 06/05/09

E-mail: dpchaves@eerp.usp.br

Aprovado: 16/06/10 


\section{INTRODUÇÃO}

O processo de trabalho na Enfermagem organiza-se em subprocessos, que podem ser denominados cuidar ou assistir, administrar ou gerenciar, pesquisar e ensinar, sendo que cada um destes possui seus próprios objetos, meios/instrumentos e atividades, coexistindo em um mesmo momento e instituição ${ }^{(1)}$.

O enfermeiro é responsável pela gerência de unidades, atividade essa que consiste na previsão, provisão, manutenção, controle de recursos materiais e humanos para o funcionamento do serviço e a gerência do cuidado, abrangendo o diagnóstico, o planejamento, a execução e a avaliação da assistência, passando pela delegação das atividades, supervisão e orientação da equipe de enfermagem ${ }^{(2)}$.

Apesar das contribuições do modelo funcional à organização do trabalho na área hospitalar, ao longo do tempo, este tem produzido efeitos negativos que prejudicam o processo de trabalho. Dentre eles, citam-se as dificuldades para responder prontamente às necessidades dos pacientes e dos trabalhadores, interferindo de forma significativa na qualidade dos serviços prestados, pois o trabalho de enfermagem é desenvolvido mecanicamente, sem maiores vínculos com os problemas cotidianos e usuários ${ }^{(3)}$.

Embora na raiz histórica da Enfermagem prevaleça o autoritarismo, centralização das decisões e impessoalidade nas relações, observa-se a introdução de novas abordagens gerenciais, em decorrência das intensas transformações que estão ocorrendo na sociedade e nas organizações de saúde, impulsionando os gerentes a buscar novas alternativas para organizar o trabalho ${ }^{(4)}$. Essas novas abordagens trazem, no seu bojo, conceitos de flexibilidade, redução da hierarquia, trabalho em equipe e descentralização das decisões, visando à satisfação dos usuários e trabalhadores, bem como à produtividade e à responsabilidade compartilhada, que devem ser vislumbradas pelos gerentes do futuro ${ }^{(4)}$.

O entrelaçamento e a aproximação entre o cuidar e o gerenciar fazem parte de um novo paradigma da Enfermagem que está sendo construído na atualidade ${ }^{(5)}$.

A supervisão de enfermagem tem potencial para aprimorar o trabalho, esperando-se que favoreça a melhoria na qualidade do cuidado, além do desenvolvimento de competências da equipe de enfermagem, por meio da educação permanente de serviço. A supervisão caracteriza-se pelo exercício de comunicação direta entre supervisor e supervisionado ${ }^{(6)}$. Essa já foi considerada uma função de caráter punitivo e fiscalizador, na qual o supervisor assumia o papel de "assegurar" o cumprimento das ordens e dos regulamentos impostos pelos níveis hierárquicos superiores, com vistas garantir o nível de produtividade ${ }^{(7)}$.

Para o exercício da supervisão na área da saúde, é necessário analisar o contexto organizacional, suas interfaces com as políticas de saúde, o acompanhamento das intervenções, respectivos resultados obtidos, o aprimoramento e qualificação dos agentes do trabalho, além de uma articulação política entre as esferas organizacionais e os profissionais da área da saúde, no intuito de promover uma assistência qualificada ${ }^{(8)}$.

Nos serviços hospitalares, nos últimos anos, especificamente na Enfermagem, o papel de supervisor vem passando por uma transformação, do estilo autoritário para democrático. Entretanto, o supervisor ainda é visto por muitos como o responsável pelo aumento da produtividade através da distribuição do trabalho entre os diferentes agentes de enfermagem, o que caracteriza a divisão social e técnica do traba1ho. Cabe destacar que o real sentido da supervisão é a orientação da equipe de enfermagem, visando o desenvolvimento e capacitação para o serviço, tendo como característica de ensino, o controle e a articulação política. Somente o enfermeiro, independente do nível hierárquico no qual atua na instituição, é responsável por desempenhar a supervisão de enfermagem baseada na coletividade e na interdependência dos diferentes trabalhadores de enfermagem ${ }^{(8)}$.

É relevante compreender o modo como o enfermeiro articula suas atividades, de modo a exercer a supervisão da equipe de enfermagem em uma perspectiva de orientação, motivação e coordenação ${ }^{(9)}$.

Dentre os inúmeros fatores associados ao processo de trabalho do enfermeiro no hospital, destacam-se os riscos ocupacionais aos quais os trabalhadores estão expostos. Riscos ocupacionais são os agentes físicos, químicos, biológicos, ergonômicos e de acidentes existentes nos ambientes de trabalho que, em função de sua natureza, concentração ou intensidade e tempo de exposição, são capazes de causar danos à saúde do trabalhador ${ }^{(10)}$. A Norma Regulamentadora de Segurança a Saúde do Trabalho em Estabelecimentos de Assistência à Saúde (NR 32) propõe a implementação de medidas para a proteção e segurança dos trabalhadores da área de saúde ${ }^{(10)}$.

Embora historicamente os trabalhadores da área da saúde nunca tenham sido considerados uma categoria profissional de alto risco para acidentes de trabalho, o risco ocupacional com agentes infecciosos 
é conhecido desde o início dos anos 40 do século XX. Entretanto, medidas profiláticas para trabalhadores expostos aos patógenos de transmissão sanguínea só foram desenvolvidas e implementadas a partir da epidemia de infecção pelo HIV/aids, no início da década de $80^{(11)}$.

O risco ocupacional para os trabalhadores da saúde é um assunto muito discutido nas últimas décadas, principalmente com enfoque na soroconversão para HIV, HBV e HCV (vírus da imunodeficiência humana, vírus da hepatite $\mathrm{B}$ e vírus da hepatite $\mathrm{C}$, respectivamente). Entretanto, os registros dos acidentes com material biológico ocorridos nas unidades de saúde não retratam a real situação ${ }^{(12)}$. Como medida de segurança à saúde dos trabalhadores, faz-se necessário o reconhecimento e a adoção de medidas preventivas, bem como o controle dos riscos, destacando o uso de equipamentos de proteção individual (EPI).

Os EPI são todos os dispositivos de uso individual, destinados a proteger a integridade física do trabalhador, incluindo luvas, protetores oculares ou faciais, protetores respiratório, aventais e proteção para os membros inferiores ${ }^{(13)}$. No Brasil, segundo recomendação do Ministério do Trabalho e Emprego, os empregadores são obrigados a fornecer os EPI adequados à minimização dos riscos aos quais os profissionais estão expostos, bem como realizar, no momento da admissão e periodicamente, programas de treinamento dos profissionais quanto à correta utilização desses equipamentos. A adequação destes EPI deve levar em consideração, não somente a eficiência necessária para o controle do risco de exposição, mas, também, o conforto oferecido; se há desconforto no uso do equipamento, existe maior possibilidade de o profissional deixar de incorporá-lo no uso rotineiro ${ }^{(14)}$.

$\mathrm{Na}$ área da saúde, dentre as medidas de prevenção adotadas na organização do trabalho, destacam-se as Precauções Padrão. Estas devem ser utilizadas pelos profissionais da saúde no cuidado a todo paciente; manuseio de artigos contaminados; contato com material biológico, como sangue, líquidos corporais, secreções e excreções (exceto suor) e mucosas. O objetivo é evitar o contato do profissional de saúde com micro-organismos do paciente ${ }^{(15)}$.

Dentre as Precauções Padrão estão elencadas recomendações relacionadas à utilização de barreiras protetoras, isto é, luvas, máscaras, aventais impermeáveis e óculos com protetores laterais, quando houver a possibilidade de contato com sangue ou outro fluido orgânico ${ }^{(16)}$. A utilização das Precauções Padrão requer nova aprendizagem, principalmente mudança de hábitos, que os profissionais com mais tempo de serviço têm dificuldade de incorporar. Alguns estudos demonstram que profissionais mais jovens e menos experientes revelam maiores níveis de utilização das Precauções Padrão ${ }^{(17)}$.

Diante do exposto, no tocante à prática gerencial do enfermeiro, particularmente a supervisão da equipe e a importância do uso de EPI, questionamos: Como o enfermeiro, responsável pela equipe de enfermagem, supervisiona a adoção de uso de equipamentos de proteção individual em um hospital geral?

A necessidade de acompanhar a utilização de EPI pelos trabalhadores de enfermagem, bem como diagnosticar possibilidades e limitações da supervisão da equipe pelo enfermeiro justificaram a realização deste estudo. Assim, o objetivo foi descrever a supervisão de enfermagem, em relação ao uso de EPI, em um Hospital Geral de um município no interior do Estado de São Paulo. Espera-se que os resultados deste estudo possam trazer contribuições para a prática gerencial do enfermeiro.

\section{MATERIAIS E MÉTODOS}

Trata-se de uma pesquisa descritiva exploratória, utilizando dados quantitativos e qualitativos. Foi desenvolvida em Ribeirão Preto, município situado na região nordeste do Estado de São Paulo, com população de 563.107 habitantes, mas que chega a 1.284.340 quando considerada a área de abrangência da Direção Regional de Saúde-XIII ${ }^{(18)}$.

Dentre os hospitais conveniados ao Sistema Único de Saúde (SUS) pela Secretaria Municipal de Saúde de Ribeirão Preto, foi considerado campo de pesquisa um hospital geral filantrópico que atende demanda espontânea e aquela referenciada pelo SUS e outros convênios, nas especialidades de clínica médica, cirúrgica e obstétrica, em regime de atenção ambulatorial e de internação. O hospital conta com um total de 153 leitos, dos quais 97 são destinados ao SUS, sendo seis leitos de UTI. O número médio de internações por mês é de 950, das quais aproximadamente 800 são de pacientes atendidos pelo SUS.

A população de estudo foi constituída por 20 enfermeiros lotados em diferentes setores do hospital e que atenderam aos seguintes critérios de inclusão: ser enfermeiro responsável pela supervisão direta da equipe de enfermagem e concordar em participar do estudo. Foram excluídos do estudos os enfermeiros 
que estavam afastados do trabalho por motivo de férias e/ou licenças.

Para coleta de dados foi utilizado um instrumento com perguntas abertas e fechadas, com três partes: caracterização do enfermeiro entrevistado quanto a idade, tempo de formação e de trabalho na instituição; condições de acesso e uso de EPI pela equipe de enfermagem a qual ele é responsável; aspectos relativos às medidas de supervisão adotadas pelos enfermeiros. Foi anexada uma folha de orientação ao sujeito de pesquisa contendo o objetivo e orientações para a participação na entrevista. O instrumento de coleta de dados foi construído especificamente para este estudo e submetido à validação aparente e de conteúdo. O grupo de validadores foi composto por três profissionais enfermeiros peritos na temática, que consideraram adequado o formato do questionário proposto. Após a validação, o instrumento foi submetido a um pré-teste, com enfermeiros que não eram sujeitos do estudo definitivo, totalizando cinco enfermeiros pós-graduandos em Enfermagem, que responderam com clareza e prontamente as questões formuladas. O tempo para o preenchimento de cada instrumento variou entre 20 e 30 minutos. A coleta de dados foi realizada nos meses de fevereiro e março de 2009.

Neste estudo, as variáveis de interesse foram: gênero, faixa etária, tempo de trabalho na Enfermagem, unidade de trabalho, tempo de serviço na unidade, turno de trabalho; quanto ao EPI, informações sobre fornecimento e treinamento para uso, disponibilidade na unidade, resistência da equipe de enfermagem ao uso, tipos usados, justificativas para o não-uso; e as medidas de supervisão de enfermagem.

Os dados quantitativos coletados foram digitados, codificados e armazenados em uma planilha eletrônica no formato Microsoft Excel. Para a análise, foi utilizada estatística descritiva, adotando-se frequências e porcentagens. Os dados qualitativos foram agrupados por similaridade de conteúdo das respostas.

O trabalho foi desenvolvido de modo a garantir o cumprimento dos preceitos da Resolução n. 196/96 sobre pesquisa envolvendo seres humanos. O projeto foi submetido à apreciação do Comitê de Ética e Pesquisa da Escola de Enfermagem de Ribeirão Preto (EERPUSP), tendo sido aprovado sob o parecer n. 0895/2008.

\section{RESULTADOS E DISCUSSÃO}

Na tabela 1, apresentamos a caracterização da população estudada quanto ao gênero, faixa etária, tempo de serviço como enfermeiro, unidade de trabalho, tempo de serviço na unidade e turno.

Sobre as características mais frequentes da população do estudo, $70 \%$ dos entrevistados são do gênero feminino; e $45 \%$ estão na faixa etária entre 20 e 30 anos. Quanto ao tempo de serviço, $40 \%$ dos participantes atuam há menos de quatro anos; $25 \%$ trabalham na supervisão de enfermagem; $65 \%$ trabalham a menos de três anos na atual unidade e $40 \%$ trabalham em turnos alternantes.

Tabela 1 - Distribuição e caracterização de enfermeiros de um hospital filantrópico. Ribeirão Preto, 2009.

\begin{tabular}{|c|c|c|}
\hline Variáveis & N. & $\%$ \\
\hline \multicolumn{3}{|l|}{ Gênero } \\
\hline Feminino & 14 & 70 \\
\hline Masculino & 6 & 30 \\
\hline Total & 20 & 100 \\
\hline \multicolumn{3}{|l|}{ Faixa etária } \\
\hline $20 \vdash 30$ anos & 9 & 45 \\
\hline $30-40$ anos & 7 & 35 \\
\hline$\geq 40$ anos & 4 & 20 \\
\hline Total & 20 & 100 \\
\hline \multicolumn{3}{|c|}{ Tempo de serviço como enfermeiro } \\
\hline$<4$ anos & 8 & 40 \\
\hline $4-8$ anos & 5 & 25 \\
\hline$\geq 8$ anos & 7 & 35 \\
\hline Total & 20 & 100 \\
\hline \multicolumn{3}{|l|}{ Unidade de trabalho } \\
\hline Supervisão & 5 & 25 \\
\hline Clínica Médica & 4 & 20 \\
\hline Centro Cirúrgico & 3 & 15 \\
\hline U.T.I. & 3 & 15 \\
\hline Ambulatório & 2 & 10 \\
\hline C.C.I.H. & 1 & 5 \\
\hline Coordenação de Enfermagem & 1 & 5 \\
\hline Faturamento & 1 & 5 \\
\hline Total & 20 & 100 \\
\hline \multicolumn{3}{|c|}{ Tempo de servico na unidade de trabalho } \\
\hline$<3$ anos & 13 & 65 \\
\hline 3 - 6 anos & 3 & 15 \\
\hline$\geq 6$ anos & 4 & 20 \\
\hline Total & 20 & 100 \\
\hline \multicolumn{3}{|l|}{ Turno de trabalho } \\
\hline Manhã & 4 & 20 \\
\hline Tarde & 4 & 20 \\
\hline Noite & 4 & 20 \\
\hline Turnos alternantes & 8 & 40 \\
\hline Total & 20 & 100 \\
\hline
\end{tabular}

É interessante enfatizar a singularidade desse 
serviço, já que possui no seu quadro $30 \%$ de enfermeiros do gênero masculino, o que pode ser considerado um alto índice, visto que a Enfermagem ainda é uma profissão predominantemente exercida por pessoas do sexo feminino.

Cabe destacar que os enfermeiros entrevistados constituem uma população eminentemente jovem, com pouco tempo de formação profissional e de inserção na unidade. Tais características podem favorecer o desenvolvimento de suas funções de supervisão da equipe, uma vez que o dinamismo característico dos jovens profissionais pode estar presente. Por outro lado, exatamente o fato ser recém-formado pode ser um fator limitante no tocante à experiência profissional na supervisão da equipe.

Segundo 90\% dos entrevistados, a Instituição fornece treinamento adequado e orientação para o uso de EPI. Em relação ao fornecimento e acesso aos EPI, 95\% afirmaram que há disponibilidade e facilidade de acesso na unidade em que trabalham. Quando questionados se há resistência da equipe de enfermagem ao uso dos equipamentos, $60 \%$ dos enfermeiros responderam que sim e $35 \%$ que não.

As falas a seguir explicam os motivos pelos quais os entrevistados acreditam que exista resistência ao uso dos equipamentos por parte da equipe de enfermagem:

Acham que não vão ter problemas de saúde; e também falta lugar adequado para guardar o equipamento. (E02)

Muitas vezes a supervisão é falha. Sempre existe a necessidade de novas orientações. (E11)

\section{Incômodo e desconforto no uso dos diferentes EPI. (E09)}

Não utilizam por pressa em resolver as coisas. Isso é consequência do estresse e falta de funcionários. (E20)

Para que as precauções sejam efetivas na prática em saúde, é necessária a adesão dos profissionais durante a realização de procedimentos assistenciais. Adesão significa manter atitudes adequadas, exigindo do profissional motivação e conhecimento técnico, porém, a relação existente entre atitude e conhecimento pode ser baixa. A falta de motivação, o deficit de conhecimento técnico da equipe, a qualificação insuficiente dos profissionais, o comportamento inadequado dos membros mais experientes, influenciando negativa- mente a equipe, e a sobrecarga de trabalhosão fatores explicativos para a baixa utilização de $\mathrm{EPI}^{(17)}$.

Quando analisada a adoção das recomendações para o uso do EPI, constata-se que muitos profissionais da saúde consideram que sua utilização prejudica o desenvolvimento das atividades profissionais. Desta forma, a perda da habilidade no desenvolvimento de tarefas, a diminuição da destreza manual, o desconforto, a inconveniência e o relato de que as luvas aumentam as chances de acidentes com agulhas ou, então, não calçam bem, são razões para não aderir ao uso ${ }^{(17)}$

A tabela 2 apresenta os EPI que são usados com mais frequência pela equipe de enfermagem.

Conforme dados da tabela 2, a máscara e as luvas são os EPI mais utilizados, sendo citados em $100 \%$ das entrevistas. Os óculos foram mencionados por $90 \%$ dos participantes; o avental/capote por $75 \%$ e $25 \%$ se referiram a outros EPI, tais como sapatos fechados $(5 \%)$, máscara N95 (10\%) e o gorro (10\%).

Tabela 2 - EPI mais usados pela equipe de enfermagem de um hospital filantrópico. Ribeirão Preto, 2009.

\begin{tabular}{lcccccc}
\hline EPI & \multicolumn{2}{c}{ Sim } & \multicolumn{2}{c}{ Não } & \multicolumn{2}{c}{ Total } \\
& N. & \% & N. & \% & N. & \% \\
\hline Máscara & 20 & 100 & 0 & 0 & 20 & 100 \\
Luvas & 20 & 100 & 0 & 0 & 20 & 100 \\
Óculos & 18 & 90 & 2 & 10 & 20 & 100 \\
Capote/Avental & 15 & 75 & 5 & 25 & 20 & 100 \\
Outros & 5 & 25 & 15 & 75 & 20 & 100 \\
\hline
\end{tabular}

O uso de EPI e a adoção das Precauções Padrão representam importantes medidas de proteção ao trabalhador. Na tabela 3, estão as justificativas dos sujeitos entrevistados para não-utilização de EPI nas unidades de trabalho.

A falta de responsabilização da equipe foi citada por $75 \%$ dos entrevistados, e $50 \%$ indicaram a diminuição da habilidade no desenvolvimento do procedimento como fator para a não-utilização. A inconveniência do uso foi citada por $40 \%$ dos entrevistados; $35 \%$ justificaram o EPI interfere no trabalho. Os itens acesso difícil aos EPI, desconhecimento de indicação Tabela 3 - Justificativas para o não uso de EPI pela equipe de enfermagem de um hospital filantrópico. Ribeirão Preto, 2009.

\begin{tabular}{lcccccc}
\hline Justificativa & \multicolumn{2}{c}{ Sim } & \multicolumn{2}{c}{ Não } & \multicolumn{2}{c}{ Total } \\
& N. & $\mathbf{\%}$ & N. & $\mathbf{\%}$ & N. & $\mathbf{\%}$ \\
\hline $\begin{array}{l}\text { Baixa responsabilização } \\
\text { da equipe }\end{array}$ & 15 & 75 & 5 & 25 & 20 & 100 \\
\hline & & & & & Continua
\end{tabular}


Continuação

\begin{tabular}{lcccccc}
\hline \multirow{2}{*}{ Justificativa } & \multicolumn{2}{c}{ Sim } & \multicolumn{2}{c}{ Não } & \multicolumn{2}{c}{ Total } \\
& N. & $\%$ & N. & $\%$ & N. & $\%$ \\
\hline $\begin{array}{l}\text { Diminuição da } \\
\text { habilidade no } \\
\text { desenvolvimento do }\end{array}$ & 10 & 50 & 10 & 50 & 20 & 100 \\
procedimento & & & & & & \\
$\begin{array}{l}\text { Inconveniência de seu } \\
\text { uso }\end{array}$ & 9 & 45 & 11 & 55 & 20 & 100 \\
$\begin{array}{l}\text { Interfere no trabalho } \\
\text { Acesso difícil aos EPI }\end{array}$ & 7 & 35 & 13 & 65 & 20 & 100 \\
$\begin{array}{l}\text { Desconhecimento de } \\
\text { indicação para o uso }\end{array}$ & 3 & 15 & 17 & 85 & 20 & 100 \\
$\begin{array}{l}\text { Falta de supervisão } \\
\text { Outros }\end{array}$ & 3 & 15 & 17 & 85 & 20 & 100 \\
& 3 & 15 & 17 & 85 & 20 & 100 \\
\hline
\end{tabular}

para o uso, falta de supervisão e outros foram citados em $15 \%$ da entrevistas em igual porcentagem.

As taxas de incidência de acidentes de trabalho por exposição a fluídos corporais e materiais perfurocortantes podem ser elevadas em relação à não-adesão às medidas de Precaução Padrão ${ }^{(19)}$.

Segundo a NR32, os EPI, descartáveis ou não, devem estar à disposição em número suficiente nos postos de trabalho, de forma que seja garantido o imediato fornecimento ou reposição, bem como deve ser garantida a sua conservação e higienização, recipientes e meios de transporte adequado para materiais infectantes, fluidos e tecidos orgânicos ${ }^{(14)}$.

Faz-se necessário que profissionais e dirigentes de serviços de saúde estejam cientes dos direitos e deveres de seguir os princípios de Precauções Padrão, utilizando medidas profiláticas que se aplicam ao sangue e demais fluídos corpóreos ${ }^{(20)}$.

Quanto às formas de supervisão adotadas pelos enfermeiros, a observação direta apareceu em 95\% das respostas; a orientação individual foi marcada em $85 \%$ das entrevistas; o item reunião e discussão em grupo foi apresentado por $80 \%$ dos entrevistados; $60 \%$ referiram a demonstração de uso e provisão de materiais com peso igual. O item 'outros' foi citado por $25 \%$, e incluía medidas como educação continuada - com $60 \%$ das respostas -, advertência verbal e a demonstração da utilização dos EPI pelo próprio enfermeiro.

A supervisão é uma atividade que faz parte do dia-a-dia do enfermeiro, pois este planeja, executa e avalia o processo de trabalho da equipe. Sendo assim, a sistematização da supervisão eleva o nível da assistência prestada à clientela, fortalece os recursos humanos e melhora os recursos materiais ${ }^{(6)}$.
A competência técnica do enfermeiro para a supervisão não é o bastante, é necessário que haja entendimento das pessoas e dos grupos para melhor coordenação dos recursos humanos. O supervisor, para integrar as pessoas no exercício de trabalho, precisa de firmeza e sensibilidade.

Quando questionados sobre as atitudes que adotam quando é percebido que um ou mais membros da equipe não utilizam os EPI ou os utilizam de forma incorreta, os enfermeiros deram sua opiniões:

Não deixo o funcionário nem começar o procedimento. Caso já tenha começado, chamo para conversar e, na persistência, faço a advertência verbal. (E17)

Re-oriento, mostro a importância do uso dos EPI. Se persistir, faço advertência oral e escrita, que é anotado na pasta do funcionário. (E12)

Questiono o porquê de não ter usado o EPI. Explico os riscos e aviso que a não-utilização dos equipamentos pode causar demissão. (E11)

Primeiro converso sobre a importância do uso do EPI. Faço discussão em grupo e, por último, uma chamada de atenção mais dura. (E20)

Advertência verbal no primeiro caso. Se houver reincidência, advertência escrita, que vai para a pasta do funcionário. (E14)

Cabe destacar que as atitudes dos enfermeiros variam desde aquelas mais focadas em medidas educativas, até as medidas mais punitivas. O papel do supervisor passa a ser de orientador e facilitador no ambiente de trabalho, quando ele agrega esforços junto à equipe para que as necessidades individuais e coletivas sejam atendidas, a fim de promover a orientação e motivação da equipe ${ }^{(9)}$. Espera-se que a supervisão seja desempenhada em uma perspectiva educativa, motivadora, conscientizadora, e não punitiva.

Os depoimentos que se seguem ilustram o papel do enfermeiro no tocante à supervisão ao uso de EPI.

Imprescindivel, para reforçar e relembrar a importância do uso dos EPI, para se proteger e proteger os pacientes. (E17)

Orientar, disponibilizar e usar os EPI corretamente. (E11) 
Em virtude da peculiaridade do trabalho do enfermeiro, este tem a atribuição de orientar/supervisionar o auxiliar/técnico de enfermagem e também de articular outras esferas administrativas para garantir o suprimento de EPI adequados para cada necessidade. É desejável que o enfermeiro incorpore às suas atividades profissionais a preocupação e o cuidado com a saúde dos trabalhadores de enfermagem sob sua supervisão.

\section{CONCLUSÃO}

Embora o hospital estudado forneça adequadamente os EPI necessários para as rotinas de trabalho, promovendo treinamentos e facilitando o acesso a estes equipamentos, existe resistência por parte da equipe em utilizá-los. As justificativas presentes no estudo vão desde aspectos relativos à responsabilidade do funcionário, até a supervisão inadequada pelo enfermeiro responsável. Quando aplicadas, as medidas de supervisão mais comuns são a de observação direta e orientação individual.

É de extrema importância que, considerando a realidade de cada serviço, os profissionais recebam treinamentos específicos para o uso dos EPI. Uma possibilidade viável é a da educação permanente, que possibilita aos enfermeiros trabalhar com seus funcionários estimulando a participação ativa dos mesmos, inclusive quando há necessidade de propor saídas para as dificuldades encontradas.

\section{REFERÊNCIAS}

1. Kurcgant P. Gerenciamento em enfermagem. Rio de Janeiro: Guanabara Koogan; 2005.

2. Greco RM. Ensinando administração em enfermagem através da educação em saúde. Rev Bras Enferm. 2004;57(4):472-4.

3. Spagnol CA. (Re) Pensando a gerência em enfermagem a partir de conceitos utilizados no campo da saúde coletiva. Ciênc \& Saúde Colet. 2005;10(1):119-27.

4. Fernandes SM., Spagnol CA, Trevizan MA, Hayashida M. A conduta gerencial da enfermeira: um estudo fundamentado nas teorias gerais da administração. Rev Latino-Am Enfermagem. 2003;11(2):161-7.

5. Rossi FR, Silva MAD. Fundamentos para processos gerenciais na prática do cuidado. Rev Esc Enferm USP 2005;39(4) 460-8.
6. Correia VS, Servo MLS. Supervisão da enfermeira em Unidade Básica de Saúde. Rev Bras Enferm. 2006;59(4):127-31.

7. Cunha KC. Supervisão em enfermagem. In: Kurcgant P, coordenadora. Administração em enfermagem. São Paulo: EPU; 1991. p.117-32.

8. Silva EM. Supervisão em enfermagem: análise crítica das publicações no Brasil dos anos 30 a década de 80 [dissertação]. Ribeirão Preto (SP): Universidade de São Paulo; 1991.

9. Liberali J. Dall'Agnol CM. Supervisão de enfermagem: um instrumento de gestão. Rev Gaúch Enferm. 2008;29(2):276-82.

10. Federação das Indústrias do Estado de São PauloFIESP. Segurança e Medicina do Trabalho. 59a ed. São Paulo: Atlas; 2006. Manuais de Legislação Atlas.

11. Ministério da Saúde (BR). Secretaria de Vigilância em Saúde. Recomendações para atendimento e acompanhamento de exposição ocupacional a material biológico: HIV e hepatites B e C. Brasília; 2004.

12. Centers for Disease Control and Prevention. Transmission of hepatitis B and C viruses in outpatient settings, New York, Oklahoma, and Nebraska 20002002. MMWR. 2003;52(38):901-6.

13. Simões M, Marques EGL, Chiarini PFT, Pires MFC. $\mathrm{O}$ uso de equipamentos de proteção individual (EPIs) e coletiva (EPCs) nos acidentes ocorridos em laboratórios de saúde pública no período de maio de 1998 a maio de 2002. Rev Inst Adolfo Luts. 2003;62(2):105-9.

14. Reis RS. Segurança e medicina do trabalho: normas regulamentadoras. São Caetano do Sul: Yendis; 2006.

15. Moura JP. A adesão dos profissionais de enfermagem às precauções de isolamento na assistência aos portadores de microorganismos resistentes [dissertação]. Ribeirão Preto (SP): Universidade de São Paulo; 2004.

16. Brevidelli MM. Modelo explicativo da adesão às precauções padrão: construção e aplicação [tese]. São Paulo (SP): Universidade de São Paulo; 2003.

17. Brevidelli MM, Cianciarulo TI. Aplicação do modelo de crenças em saúde na prevenção dos acidentes com agulhas. Rev Saúde Pública. 2001;35(2):193-201.

18. Ministério da Saúde (BR). População residente - 
Estimativas para o TCU - São Paulo [Internet]. Brasília: DATASUS. [acesso em 09 set 2008]. Disponível: http:// tiny.cc/rl2pr

19. Lopes ACS, Oliveira AC, Silva JT, Paiva MHRS. Adesão às precauções padrão pela equipe do atendimento préhospitalar móvel de Belo Horizonte, Minas Gerais, Brasil. Cad Saúde Pública. 2008;24(6):1387-96.

20. Ribeiro AS, Gabatz RIB, Neves ET, Padoin SMM. Caracterização de acidente com material perfurocortante e a percepção da equipe de enfermagem. Cogitare Enferm 2009; 14(4):660-6. 